\title{
Calibration of Temperature Sensors with Different Tolerances for Polymerase Chain Reaction Chips
}

\author{
Chan-Young Park, ${ }^{1,2}$ Seul-Bit-Na Koo, ${ }^{1,2}$ Jae-Hyeon Cho, ${ }^{1,2}$ Yu-Seop Kim, ${ }^{1,2}$ \\ Hye-Jeong Song, ${ }^{1,2}$ and Jong-Dae Kim ${ }^{1,2 *}$ \\ ${ }^{1}$ Department of Convergence Software, Hallym University, \\ 1 Hallymdaehak-gil, Chuncheon-si, Gangwon-do 24252, Korea \\ ${ }^{2}$ Bio-IT Research Center, Hallym University, 1 Hallymdaehak-gil, Chuncheon-si, Gangwon-do 24252, Korea
}

(Received February 26, 2016; accepted February 27, 2017)

Keywords: micro-PCR chip, calibration, thermistor, resistance tolerance, PCR performance

The calibration of temperature sensors with different tolerances is investigated to evaluate the effect of a polymerase chain reaction (PCR) on the performance of a PCR chip. Two types of thermistor sensors with 0.5 and $1 \%$ tolerances are applied to printed circuit board (PCB)-based PCR chips. The PCR performance is evaluated for calibrated and uncalibrated thermistors. The band volume variation of gel images is analyzed statistically to evaluate the performance. As a result of analyzing the band volume in gel images, the $0.5 \%$ thermistor was found to exhibit a level of performance that is almost equal in both the calibrated and uncalibrated thermistors.

\section{Introduction}

The polymerase chain reaction (PCR) is a technology that enables the selective amplification of specific DNA fragments for very small amounts of DNA. PCR inspection is used in the diagnosis of many diseases. However, current commercial PCR equipment is both large and costly. ${ }^{(1-3)}$ Labon-a-chip PCR thermal cyclers that use micro-electromechanical systems (MEMS) are currently being developed in the gene analysis field as a means of overcoming these problems. ${ }^{(3,4)}$ They incorporate microfluidic channels and are commonly referred to as 'PCR chips. ${ }^{\text {(4-7) }}$ PCR chips obtain information rapidly through the gene analysis of extremely small quantities of DNA, and thus can be used to diagnose diseases. ${ }^{(8-11)}$ The most important function of PCR chips is accurate temperature control. ${ }^{(12,13)}$ However, research into their degree of accuracy has been limited. Temperature is the most important factor affecting successful PCR amplification. Effective temperature control can reduce reagent consumption by miniaturizing the systems and limiting the analysis time within a given cycle. This can also enhance the portability of the equipment. ${ }^{(13)}$

We previously selected a negative-temperature-coefficient (NTC) thermistor ${ }^{(14,15)}$ with a $1 \%$ resistance error as the temperature sensor. At that time, we reported a comparison of the PCR performance according to the temperature calibration of the sensor. ${ }^{(16)}$ In that study, we compared the band volume results from electrophoresis photos after DNA amplification. The $t$-test and ranksum test results for the band volumes of the calibrated and uncalibrated chips were 0.45 and 0.41 , respectively, showing that the difference in the PCR performance is insignificant. However, we concluded that calibration was advantageous from a visual inspection of the statistical box plot.

*Corresponding author: e-mail: kimjd@hallym.ac.kr

http://dx.doi.org/10.18494/SAM.2017.1548 
In that study, the channel of the PCR chips was constructed using polymer film and double-sided tape on a printed circuit board (PCB) substrate. The PCR chip was heated and cooled with a PCB heater pattern and fan, respectively, and its temperature was measured with an NTC-thermistor sensor mounted under the PCB. The chips have since been further developed as they cost less to manufacture than conventional silicon or glass-based PCR chips.

In this study, we investigated the difference in the level of performance according to the calibration of the PCR chip when using a more accurate temperature sensor than that used in the previous work. In this study, we used thermistors with a $0.5 \%$ tolerance. As in the previous study, thermistors with different degrees of error were selected and the amplifications that could be attained before and after the calibration were compared.

The materials and experimental methods are described in Sect. 2. The band volumes obtained from the electrophoresis images are compared in Sect. 3. We conclude this paper in Sect. 4.

\section{Materials and Methods}

\subsection{Chip structure}

The micro-PCR chip used in this study has a 4-layer structure, as shown in Fig. 1(a). The chip was manufactured using the same type of polypropylene (PP) as is commonly used for producing the tubes used in laboratories. To attain a constant capacity and prevent bursts, the layers of the chip were bonded using a laminator. PP box tape was used to cover the top surface of the PCB substrate to prevent any adsorption. On the box tape, $400-\mu \mathrm{m}$ double-sided tape was used to form the wall of the chamber and secure the reservoir space. The difficulty with inserting the reagent owing to the straightness of the chamber was alleviated by adopting a curved shape. As a result, the reagent could be inserted more smoothly, while bubbling was reduced within the chamber. Figure 1(b) shows the PCB together with the heater pattern and thermistor. The heating pattern is used to heat the reagent. The NTC thermistor sensor was mounted in the center of the heating pattern to measure the chamber temperature.

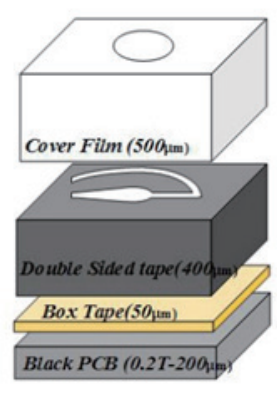

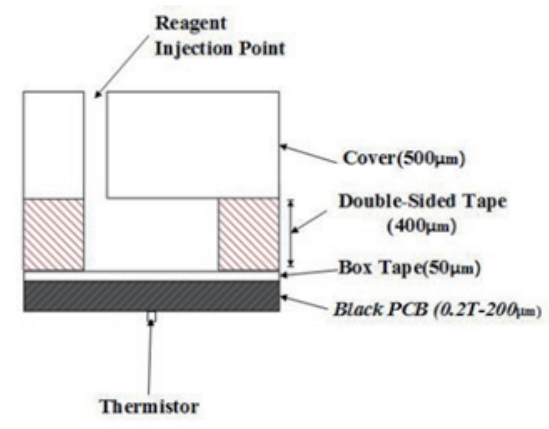

(a)

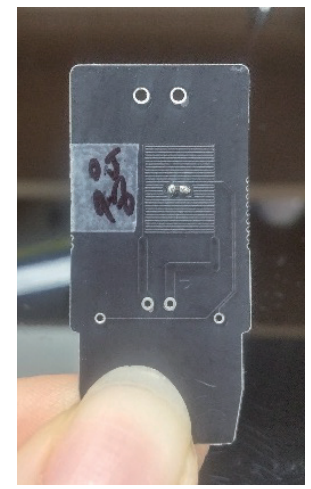

(b)

Fig. 1. (Color online) Structure of chip: (a) chamber layer structure and (b) bottom of PCR chip. 


\subsection{PCR chip controller}

The chip controller features a local-host configuration to interconnect a personal computer (PC) and the local systems. This was implemented with a microcontroller through a USB connection. For the local systems, a microcontroller (PIC18F4553) that incorporates an analog-to-digital converter (ADC) and pulse width modulator (PWM) was selected to control the PCR chips. The resistance of the NTC thermistor sensor attached to the chip was converted by the ADC and then sent to the host to be converted to a temperature value. This was compared with the target temperature, and then a PWM value was determined to control either the heater pattern or the fan. This value is determined using a proportional-integral-derivative control method and is then sent back to the local system. The local system uses this PWM value to control the heater or fan. The host PC manages the protocol files and controls the graphical user interface (GUI) in addition to the temperature and PWM calculations.

\subsection{Experimental setup}

In this experiment, NTC-thermistors (NCP15XH103D03RC, Murata Manufacturing Co., Ltd.) with a $0.5 \%$ tolerance and a resistance of $10 \mathrm{k} \Omega$ at $25^{\circ} \mathrm{C}$ were used. The measured resistance was converted to the current thermistor temperature. The temperature with respect to resistance was determined using the 3-parameter Steinhart-Hart (SH) equation. The three calibrated coefficients were calculated from the resistance-temperature pairs at four temperature points, namely, 50, 60, 72 , and $95^{\circ} \mathrm{C}$, as measured in a constant-temperature water bath. The default SH coefficients for the uncalibrated thermistors were determined by extracting the resistance-temperature pairs from the datasheet provided by the supplier.

The denaturation, annealing, and extension temperatures were 95,60 , and $72{ }^{\circ} \mathrm{C}$, respectively. The highest denaturation temperature was the most critical to the temperature error, and 16 thermistors that represented thermistor variation were selected by measuring their resistances at 95 ${ }^{\circ} \mathrm{C}$.

As listed in Table 1, four values corresponding to the highest temperature, four corresponding to the lowest temperature, and eight closest to the mean temperature in the water bath at $95{ }^{\circ} \mathrm{C}$

Table 1

Classifications of thermistors with measured temperatures at $95{ }^{\circ} \mathrm{C}$ and corresponding thermistor identification.

\begin{tabular}{lcc}
\hline Class & Temperature $\left({ }^{\circ} \mathrm{C}\right)$ & $\begin{array}{c}\text { Corresponding } \\
\text { thermistor ID }\end{array}$ \\
\hline & 97.6979 & 54 \\
$\mathrm{H}$ & 95.574 & 19 \\
& 95.5565 & 48 \\
& 95.5531 & 80 \\
$\mathrm{~L}$ & 94.9288 & 96 \\
& 95.0006 & 32 \\
& 95.0657 & 7 \\
& 95.076 & 102 \\
$\mathrm{M}$ & 95.3379 & 29 \\
& 95.3413 & 72,88 \\
& 95.3448 & 17 \\
\hline & 95.324 & 65,97 \\
\hline
\end{tabular}


were selected and classified as the ' $H$ ', 'L', and ' $M$ ' classes, respectively. We employed four controllers to minimize the variations in the biochemical process, as shown in Fig. 2. To alleviate the between-controller variation, four experiments were performed, and all of the classes were evenly assigned to each controller as listed in Table 2. The systems shown in Fig. 2 were vertically aligned and were numbered from the bottom layer to the top layer.

PCR amplification processes were performed twice for each experiment: the first applied the corrected coefficients while the other applied the default coefficients. The PCR products obtained with the corrected coefficients were named the 'calibrated amplicons' while those obtained with the default coefficients were named the 'uncalibrated amplicons'. Electrophoresis was performed on all of the eight amplicons resulting from each experiment.

\subsection{Biochemical protocol}

The reagent used for PCR was comprised of $1 \mathrm{ng} / 15.84 \mu \mathrm{l}$ of DNA (Chlamydia trachomatis), 18 $\mu 1$ of master mix, $10 \mathrm{pM} / 0.72 \mu 1$ of primer $\mathrm{F}, 10 \mathrm{pM} / 0.72 \mathrm{ml}$ of primer R, and $10 \mathrm{pM} / 0.72 \mu 1$ of probe (total $36 \mu \mathrm{l}$ ). Each cycle was comprised of three stages: denaturing at $95{ }^{\circ} \mathrm{C}$ for $10 \mathrm{~s}$, annealing at 60 ${ }^{\circ} \mathrm{C}$ for $30 \mathrm{~s}$, and extension at $72{ }^{\circ} \mathrm{C}$ for $30 \mathrm{~s}$. Each PCR run began with a hot start at $95{ }^{\circ} \mathrm{C}$ for 3 min and ended with a final extension at $95{ }^{\circ} \mathrm{C}$ for $10 \mathrm{~s}$ and at $50{ }^{\circ} \mathrm{C}$ for $30 \mathrm{~s}$. The calibrated and uncalibrated amplified results obtained from each experiment were loaded into $2 \%$ agarose gel for electrophoresis for $25 \mathrm{~min}$ at $135 \mathrm{~V}$. A commercial digital camera (EOS 450D, Canon) was used for UV filming. The camera aperture was set to 5.6, the ISO value to 1600 , and the focal length to $42 \mathrm{~mm}$ to enable comparison of the gels with the same brightness.

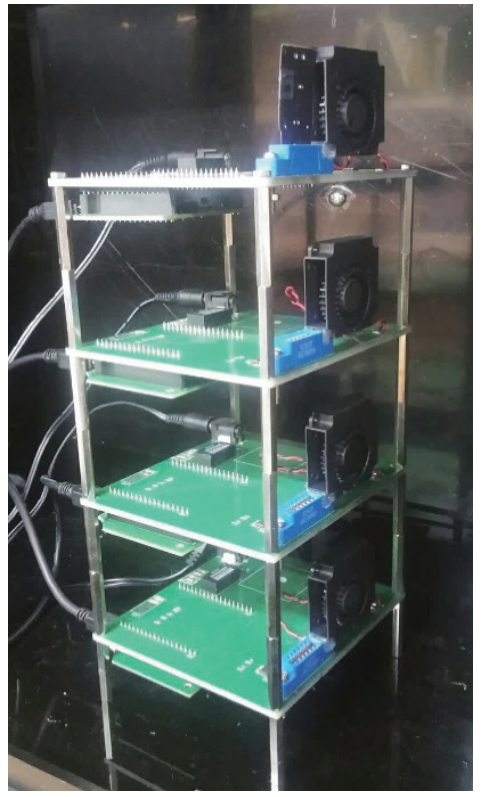

Fig. 2. (Color online) Setup of the four chip-controllers.
Table 2

Chip class assignment for each system in the four experiments to minimize the between-controller variation.

\begin{tabular}{lcccc}
\hline $\begin{array}{l}\text { System } \\
\text { No. }\end{array}$ & Exp. 1 & Exp. 2 & Exp. 3 & Exp. 4 \\
\hline 1 & $\mathrm{H}$ & $\mathrm{M}$ & $\mathrm{M}$ & $\mathrm{L}$ \\
2 & $\mathrm{~L}$ & $\mathrm{H}$ & $\mathrm{M}$ & $\mathrm{M}$ \\
3 & $\mathrm{M}$ & $\mathrm{L}$ & $\mathrm{H}$ & $\mathrm{M}$ \\
4 & $\mathrm{M}$ & $\mathrm{M}$ & $\mathrm{L}$ & $\mathrm{H}$ \\
\hline
\end{tabular}




\section{Results}

Figure 3 shows a stack of four gel images. The topmost gel image is that for the first experiment; the second image is for the second experiment, and so on. The ladders are shown in the first and last column of each image. The even and odd columns indicate the bands of uncalibrated amplicons and calibrated amplicons, respectively.

GelAnalyzer (www.gelanalyzer.com) was used to analyze the band brightness. As shown in Fig. 3, the background luminance changes vertically, so the rolling ball algorithm is applied before the band detection to detect and compensate for changes in the background (ball radius of 250 pixels). Figure 4 shows an example of an analysis for the seventh column of the third gel image. The upper graph in the figure shows the overlapped brightness profile and rolling ball trajectory. The red lines in the lower image show the peak and ridge areas used to calculate the band volume.

The values of extracted band volume are summarized in Table 3. The column number is given in the first row of the table and the band volumes are sequentially summarized starting from the second row of the first experimental result. The first and tenth columns show the band volumes of the ladder bands for which the migration distances are closest to those of the amplicons. As shown in Fig. 3, the ladder brightness varies between gel images. Therefore, the band volume of each amplicon was normalized with the average for the ladder bands shown in the first and tenth columns of Table 3. The normalized band volumes were rearranged according to whether they were calibrated or uncalibrated, as summarized in Table 4. An excessively low brightness

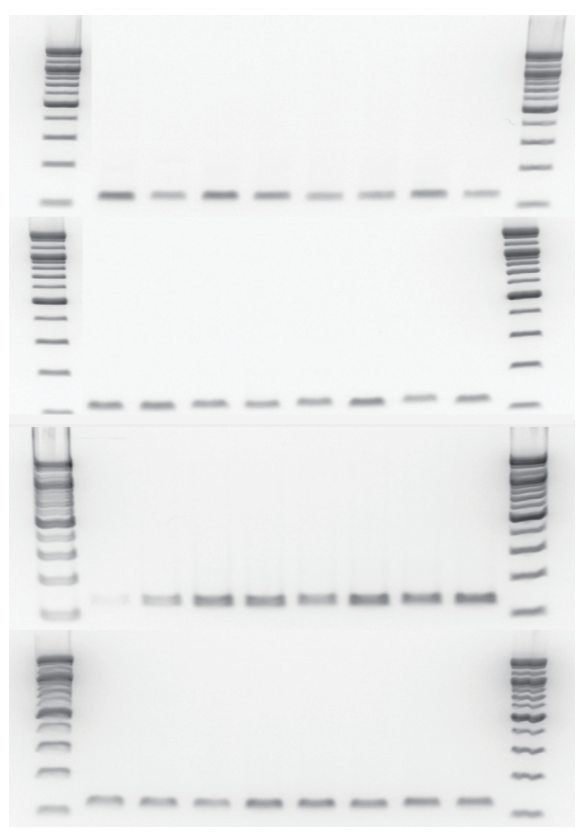

Fig. 3. (Color online) Gel images for all experimental results. The odd columns correspond to the calibrated amplicons and the even columns are for the uncalibrated amplicons excluding the ladders on both sides.

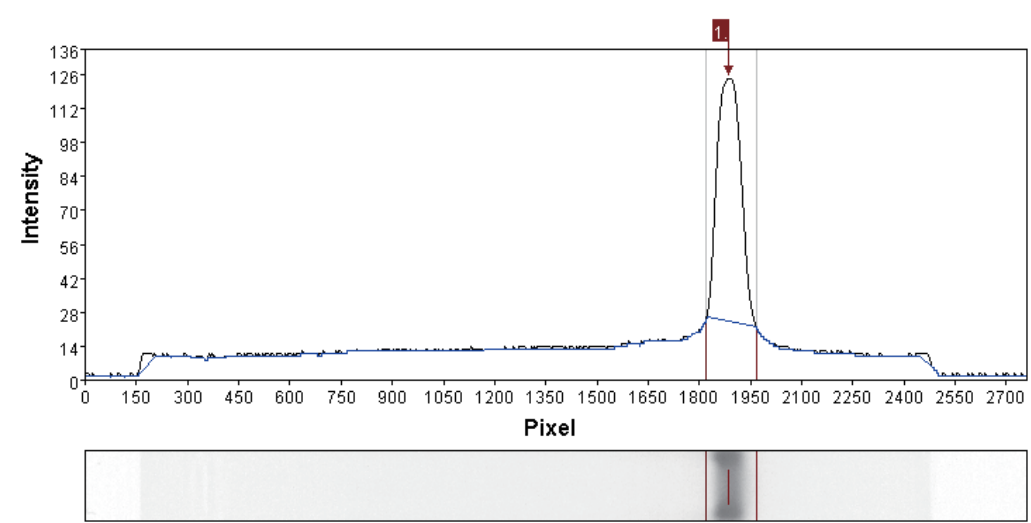

Fig. 4. (Color online) Band detection example using GelAnalyzer. Upper graph: profile of seventh column of third gel image shown in Fig. 3. Lower image: the extracted lane image and band volume calculation area (red lines). 
Table 3

Calculated band volume using GelAnalyzer (first and tenth columns depict the band volumes of one of the ladder bands for which the migration distance to the amplicons is shortest).

\begin{tabular}{ccccccccccc}
\hline & 1 & 2 & 3 & 4 & 5 & 6 & 7 & 8 & 9 & 10 \\
\hline 1 & 2767 & 5983 & 3078 & 6005 & 4353 & 2736 & 3117 & 4645 & 2549 & 2560 \\
2 & 2069 & 4568 & 4807 & 3858 & 3261 & 3666 & 5373 & 2834 & 4014 & 2946 \\
3 & 1499 & 211 & 2342 & 6700 & 6466 & 4959 & 8286 & 6892 & 8256 & 4487 \\
4 & 2562 & 3650 & 3854 & 3298 & 5634 & 5373 & 4737 & 4963 & 4998 & 3312 \\
\hline
\end{tabular}

Table 4

Relative band volumes normalized by ladder volumes.

\begin{tabular}{lcccccccc}
\hline \multicolumn{8}{c}{ Normalized band volumes } \\
\hline 1 & 2.25 & 2.25 & 1.03 & 1.74 & 1.16 & 1.63 & 1.17 & 0.96 \\
2 & 1.82 & 1.54 & 1.46 & 1.13 & 1.92 & 1.30 & 2.14 & 1.60 \\
3 & 0.07 & 2.24 & 1.66 & 2.30 & 0.78 & 2.16 & 2.77 & 2.76 \\
4 & 1.24 & 1.12 & 1.83 & 1.69 & 1.31 & 1.92 & 1.61 & 1.53 \\
\hline Mean & \multicolumn{3}{c}{1.59} & \multicolumn{5}{c}{1.67} \\
Std dev. & 0.59 & \multicolumn{5}{c}{0.58} \\
\hline
\end{tabular}

was observed for the band in the second column of the third experiment, as shown in Fig. 3. In addition, it was clear that the normalized volume, listed in Table 4, fell outside a multiple of the standard deviation for the remaining average. Therefore, this PCR amplification was judged to have failed and the statistics were calculated by replacing this value with the average of the remaining normalized volumes for the uncalibrated results. The means and the standard deviations obtained in this manner are depicted in the last two rows of Table 4.

There was no significant difference in the average band volume of the calibrated and uncalibrated results. This was also clear from the box plot shown in Fig. 5, in which our previous results, obtained with the $1 \%$ tolerance thermistors, are shown together for comparison. A visual inspection of the $0.5 \%$ boxplot does not reveal any difference between the calibrated case $(0.5 \%$ cal) and uncalibrated case $(0.5 \%$ uncal). In contrast, we can see from the box plots that the calibration is safer for a $1 \%$ tolerance thermistor.

The $p$-values for the $t$-test and rank-sum test were 0.6857 and 0.9850 , respectively, both of which were greater than the values obtained in the previous work using a $1 \%$ tolerance thermistor $(1 \% t$-test $p$-value $=0.45$, rank-sum test $p$-value $=0.41)$. These results also indicate that there is no proof that the uncalibrated use of the $0.5 \%$ tolerance thermistors is unsafe.

\section{Discussion and Conclusions}

PCR performance was investigated to observe the effect of thermistor calibration. The $1 \%$ tolerance thermistor did not prove significant in the previous study, although we concluded that calibration would be effective. In this study, the calibration effect was investigated using an NTC thermistor with a tolerance of less than $0.5 \%$.

The results of the $t$-test and rank-sum test on the calibrated and uncalibrated $0.5 \%$ tolerance chip exhibited an insignificant $p$-value. Higher $p$-values than those used with the $1 \%$ tolerance 


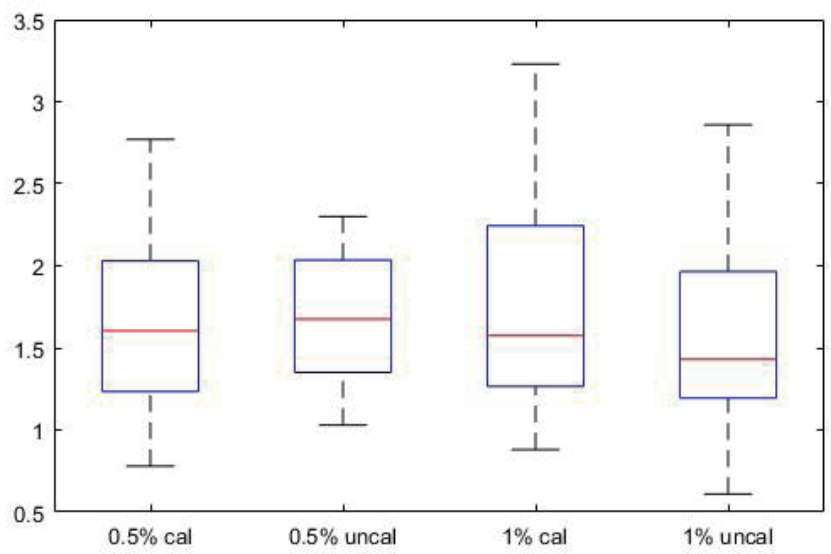

Fig. 5. (Color online) Box plot of band volumes listed in Table 4; box plots for $1 \%$ tolerance thermistors used in our previous work are also shown for comparison.

thermistor allowed us to conclude that there was no calibration gain for the PCR performance, as was observed in the previous study. The visual inspection of the statistical box plots also suggested that the uncalibrated use of $0.5 \%$ tolerant thermistors contrasts to the case of $1 \%$ tolerant thermistors.

Based on these results, it is clear that calibration is not required in the $0.5 \%$ case. The $0.5 \%$ tolerance thermistors are more expensive than the $1 \%$ thermistors. However, the calibration cost will be an important factor in the mass production of PCR chips.

\section{Acknowledgments}

This material is based upon work supported by the Ministry of Trade, Industry \& Energy (MOTIE, Korea) under Industrial Technology Innovation Program No. 10052106, 'Development of CMOS/ MEMS hybrid biosensor array platform'.

\section{References}

1 A. J. de Mello: Lab Chip 1 (2001) 24N.

2 D. Patko, Z. Martonfalvi, B. Kovacs, F. Vonderviszt, M. Kellermayer, and R. Horvath: Sens. Actuators, B 196 (2014) 352.

3 Y. S. Shin, K. Cho, S. H. Lim, S. Chung, S.-J. Park, C. Chung, D.-C. Han, and J. K. Chang: J. Micromech. Microeng. 13 (2003) 768.

4 M. Focke, D. Kosse, C. Müller, H. Reinecke, R. Zengerle, and F. von Stetten: Lab Chip 10 (2010) 1365.

5 J. D. P. Nath, T. S. Maity, F. Pettersson, J. Resnick, Y. Kunde, N. Kraus, and N. Castano: Adhesive Transfer Tape Options for Microfluidic Polymerase Chain Reaction - Solvent Free, Reel-to-Reel Compatible, Rapid Prototyping for Lab-on-a-Chip Applications, Report No. LA-UR-11-11286 (2011).

6 H. B. De Torres, C. Rensch, M. Fischer, A. Schober, M. Hoffmann, and J. Muller: Sens. Actuators, A 160 (2010) 109.

7 C. Y. Park, J. D. Kim, J. H. Ku, Y. S. Kim, H. J. Song, and J. Kim: Sens. Lett. 10 (2012) 1197.

8 C. Koo, M. Malapi-Wight, H. S. Kim, O. S. Cifci, V. L. Vaughn-Diaz, B. Ma, S. Kim, H. Abdel-Raziq, K. Ong, Y. K. Jo, D. C. Gross, W. B. Shim, and A. Han: PLoS One 8 (2013) e82704. 
9 V. Venkataraman, K. K. Kumar, R. P. Jayaraman, Sankaranand K. Narasimha, R. M. Radhakrishnan, S. Viswanaihan, C. B. Nair, P. V. Subbarao, M. Jagannath, S. Chennakrishnaiah, and S. Mondal: Hand-Held Micro PCR Device, Patent No. US20100297640 A1 (2010).

10 D. S. Lee, S. H. Park, K. H. Chung, and H. B. Pyo: IEEE Sens. J. 8 (2008) 558.

11 D. Moschou, N. Vourdas, G. Kokkoris, G. Papadakis, J. Parthenios, S. Chatzandroulis, and A. Tserepi: Sens. Actuators, B 199 (2014) 470.

12 C. Park, S. Lee, Y. Kim, H. Song, and J. Kim: Sens. Mater. 28 (2016) 593.

13 K. Shen, X. Chen, M. Guo, and J. Cheng: Sens. Actuators, B 105 (2005) 251.

14 J. Howes: J. Br. Inst. Radio Eng. 13 (1953) 228.

15 Spectrum Sensors \& Controls, Inc.: NTC Thermistor Engineering Notes, www.SpecSensors.com (2005).

16 S. B. N. Koo, J. H. Cho, Y. S. Kim, H. J. Song, C. Y. Park, and J. D. Kim: Int. J. Control Autom. 8 (2015) 149. 